\title{
ORTHOGONAL TRANSFORMATION IN EXTRACTING OF COMMON MODE ERROR FROM CONTINUOUS GPS NETWORKS
}

\author{
Maciej GRUSZCZYNSKI *, Anna KLOS and Janusz BOGUSZ
}

Faculty of Civil Engineering and Geodesy, Military University of Technology, 00-908 Warsaw, Kaliskiego St. 2, Poland

*Corresponding author's e-mail: maciej.gruszczynski@wat.edu.pl

\begin{tabular}{l} 
ARTICLE INFO \\
\hline Article history: \\
Received 24 January 2016 \\
Accepted 10 April 2016 \\
Available online 27 April 2016 \\
\hline
\end{tabular}

\section{Keywords:}

GPS

Empirical Orthogonal Function Principal Component Analysis Noise analysis

\begin{abstract}
Common Mode Error (CME) means the sum of environmental and technique-dependent systematic errors in GPS position time series. The CME, which is a kind of the temporally correlated noise, can be seen in the time series from regional GNSS networks that spans hundreds of kilometers. This paper concerns the results of studies regarding the necessity of spatio-temporal filtration of time series to determine highly reliable velocities of permanent stations for the geophysical (plate motion or earthquakes) studies or to maintain the kinematic reference frames. In this research the JPL (Jet Propulsion Laboratory) PPP solution processed by GIPSY-OASIS software were taken. Trend and seasonal signals were removed using leastsquares estimation to form the residual time series. Then, the internal structure (CME) of the set of residual time series with orthogonal transformations was revealed. We examined the Principal Component Analysis (PCA) and assumed the existence of a non-uniform spatial response in the network to the CME. We confirmed our theoretical assumptions about the benefits of the PCA approach when stations in a network are potentially affected by local effects. We noticed for height time series, that noise amplitudes decreased from 0.5 to $13.5 \mathrm{~mm} / \mathrm{year}^{-\mathrm{k} / 4}$ after filtration. That gave a relative reduction of amplitudes ranging between $4 \%$ and $76 \%$ for all stations, while the average improvement was $49 \%$. The average relative increase of spectral index was equal to $48 \%$. One of the most important consequences related to spatio-temporal GNSS time series filtering is improvement (better credibility) of accuracy of the determined velocity. The accuracy of velocity, after filtration, was lower for Up component of all stations. The average reduction was $0.2 \mathrm{~mm} /$ year, while maximal reached $0.8 \mathrm{~mm} /$ year. Above described result mean that the reduction of accuracy relative to the after-filtration accuracy was on average about $70 \%$.
\end{abstract}

\section{INTRODUCTION}

The use of the term "network," in respect to geodetic measurements, implies the existence of relationships between control points by means of common observations. The term "GPS station network" is primarily used to describe two different scenarios. The one scenario refers to a network of stations, for which data is processed in a specific way (network solution - NS), in order to achieve a common purpose, for example to maintain geodetic datum (e.g. EPN - EUREF Permanent Network). In this paper, we use an alternative meaning (stations affected by the same errors) of GPS networks. Detrending and de-seasonalizing of topocentric GPS time series resulted in residuals, which we further analyzed. These residuals are spatially and temporally correlated (e.g. Santamaría - Gómez et al., 2011; Shen et al., 2013; Bogusz et al., 2015). This assumption is the basic premise of the existence of Common Mode Errors (CME) in coordinate time series, obtained at permanent stations located less than $2000 \mathrm{~km}$ from each other (Wdowinski et al., 1997; Márquez-Azúa and DeMets, 2003; Amiri-Simkooei, 2013). The above mentioned network size was determined to be a maximum, where $\mathrm{CME}$ can be observed and considered as a uniform value. However, this is not strictly defined. The maximum network size depends on many factors (Wdowinski et al., 1997). The most significant factor is the geographic location of a network and the subsequent impact of various physical, spatially correlated error sources (Mao et al., 1999; Nikolaidis, 2002). The potential CME sources that similarly affect stations' coordinates are (Wdowinski et al., 1997; Nikolaidis, 2002; Dong et al., 2006; He et al., 2015; Tian and Shen, 2016):

1. reference frame realization, including the mismodeling of Earth Orientation Parameters (EOP);

2. error sources related to satellites, which are usually visible simultaneously in small networks 
- mismodeling of satellite: orbits, clocks, or antenna phase center corrections;

3. the unmodeling of large-scale atmospheric and hydrologic effects, as well as small scale crust deformations;

4. systematic errors caused by algorithms, software, and data processing strategies.

The necessity of CME filtration on continuous GPS time series was first identified in literature by Wdowinski et al. (1997). It is generally recognized, that GNSS residuals are a combination of types of noise, such as white, flicker, and random walk (e.g. Mao et al., 1999; Williams, 2003; Williams et al., 2004; Kenyeres and Bruyninx, 2009). The use of the "stacking" method for CME extraction resulted in the reduction of noise amplitudes for different kinds of noises. The stacking approach assumes a uniform spatial response to $\mathrm{CME}$ sources, and therefore the value of CME is the same for all stations in a network over a corresponding epoch. This approach was extended by Nikolaidis (2002), who demonstrated that CME calculations will be incorrect when station coordinates are not of the same accuracy. This method called "weighted stacking" takes into account standard errors of each coordinate, as weights (Teferle et al., 2002; Li et al., 2015). The above mentioned method gives reliable results for networks with stations spaced up to $500 \mathrm{~km}$ apart (Wdowinski et al., 1997).

Another method for CME-substraction is spatial filtering, also commonly labeled as "stacking". This method is distinguished by the fact, that spatial filters give "better fitted" CME values for individual stations, but unequal values over the entire network. As a result, spatial filters can reveal some weak local effects, treating them as common signals. Spatial filters were previously used i.e. by Márquez-Azúa and DeMets (2003), with weights depending on two factors: the length of the time series and distance between stations being considered. Another case was presented by Tian and Shen (2011), with correlation coefficients taken into consideration. More complex methods are KLE (Karhunen-Loève Expansion) and PCA (Principal Component Analysis), which implement Empirical Orthogonal Functions (EOF) to reveal common signals in residual time series (Dong et al., 2006) or to uncover signals related to regional tectonic movements (Savage, 1995; Tiampo et al., 2004). PCA is a statistical procedure that uses orthogonal transformation to reveal the internal structure of data, which is CME (Jackson, 1991; Williams et al., 2004). The proper application of this method, allows to identify CME, as a first principal component (PC) or the linear combination of the leading principal components. Another instance where PCA can be applied, is when determining the deformation (vector) field on the crustal surface, when the density of stations on a network is high (Chang and Chao, 2014). The advantage of decomposition into EOFs, is that these methods are applicable to larger networks, because CME is "regionally adjusted" for individual stations. Moreover, the signals can be separated, which allows to test the statistical significance, thus preventing insignificant signals from being included in CME calculations. This ensures that the CME value is more reliable. These methods were modified in order to increase the reliability of CME extraction with gaps in the data (Shen et al., 2013), as well as to take into account formal coordinate errors ( $\mathrm{Li}$ et al., 2015).

In this paper, we propose to apply PCA on a set of European stations. We extract the Common-Mode Error by subtracting the first component, which accounts for $40 \%$ of data variance. This way we can obtain a filtered time series, which then can be analyzed using Maximum Likelihood Estimation (MLE). We proved that not only the RMS of data is reduced, as it was previously shown by Shen et al. (2013) or Li et al. (2015), but also the stochastic part gets closer to white noise. We present hard numbers that emphasize the need of spatio-temporal filtration and reduction of flicker noise. This paper contributes to previously published analyses with a description of noise character of filtered residuals. Therefore, one becomes aware of how significant of an impact spatiotemporal filtering has on GPS time series in terms of spectral index and amplitudes of power-law process.

\section{DATA AND METHOD}

In this research, we used time series expressed in the IGS08 (Rebischung et al., 2012), provided by JPL (Jet Propulsion Laboratory). GPS processing was performed using the GIPSY-OASIS software in PPP (Precise Point Positioning) mode. It has already been recognized that the PPP processing is a contributing factor on final results being less spatially correlated (Witchayangkoon, 2000). In contrast, the network solution is more susceptible to these kind of errors. In order to form a GPS station network that meets the demands of a significant common spatial response to the CME, we chose 87 stations located in Central Europe (Fig. 1). The distance between any two stations is less than $1900 \mathrm{~km}$. Daily sampled coordinate time series do not start and stop at the same time and they have few data gaps. In the lower part of the Figure 1 we can see how many stations provided coordinates in a corresponding epoch. In other words, we can see on the vertical axis how many station's coordinates we can take into account at a given epoch from set of 87 time series taken to analysis. In spatiotemporal filtering approaches, a large number of observations need to be carried out at the same time, in order to achieve reliable results (Shen et al., 2013). In order to perform spatio-temporal filtering, we limited data timespan to a range between 2002.65 and 2011.83, while the shortest time series are more than 4 years long.

In order to detect and remove outliers, we adopted the 1D signal Hampel filter, which is a moving window implementation of the Hampel identifier (Liu et al., 2004). Sequential T-test Analysis 

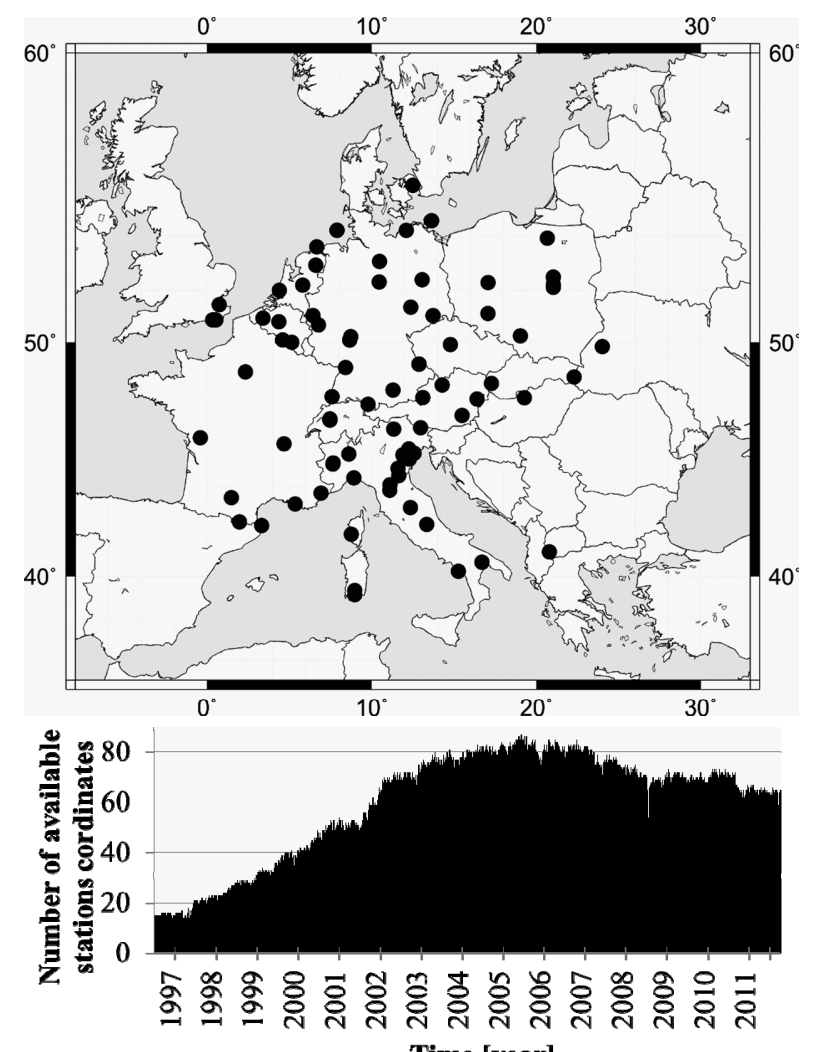

Time [year]

Fig. 1 Location of the 87 GPS stations that form a network potentially affected by CME (top) and number of stations coordinates available at a corresponding epoch - data timespan (bottom).

of Regime Shifts (STARS) algorithm (Rodionov and Overland, 2005) was applied to detect epochs of offsets.

Time series taken from stations located on the same tectonic plate, and similarly affected by geophysical processes, are highly correlated due to comparable linear trends and periodicities. Trend and seasonal signals represent the deterministic part of a time series, while residuals are the remainder of the modeling process. Residuals should reflect the stochastic process, but instead of noise, we have to assume a uniform temporal function across the network (as it was assumed at the beginning). In our research, the deterministic part was modeled using the Least Squares method, where the initial value $x_{0}$, linear trend $v_{x} \cdot t$ and periodicities $A \cdot \sin (\omega \cdot t+\varphi)$ were included in the equation. Periodicities are composed of: fortnightly and 1st through 9th harmonic of Chandler, tropical, and draconitic oscillations. The equation describing each of the components (North, East and Up) of topocentric time series is:

$$
\begin{aligned}
& x(t)=x_{0}+v_{x} \cdot t+A^{13.66} \cdot \sin \left(\omega^{13.66} \cdot t+\varphi^{13.66}\right) \\
& +A^{14.6} \cdot \sin \left(\omega^{14.6} \cdot t+\varphi^{14.6}\right) \\
& +A^{14.19} \cdot \sin \left(\omega^{14.19} \cdot t+\varphi^{14.19}\right) \\
& +A^{14.76} \cdot \sin \left(\omega^{14.76} \cdot t+\varphi^{14.76}\right) \\
& +\sum_{i=1}^{9}\left[A_{i}^{C H} \cdot \sin \left(\omega_{i}^{C H} \cdot t+\varphi_{i}^{C H}\right)\right] \\
& +\sum_{i=1}^{9}\left[A_{i}^{T} \cdot \sin \left(\omega_{i}^{T} \cdot t+\varphi_{i}^{T}\right)\right] \\
& +\sum_{i=1}^{9}\left[A_{i}^{D} \cdot \sin \left(\omega_{i}^{D} \cdot t+\varphi_{i}^{D}\right)\right]+\underbrace{[\mathbf{C M E}(t)+\mathbf{\varepsilon}(t)]}_{\mathbf{r}(t)}
\end{aligned}
$$

where the superscripts $\mathrm{CH}, \mathrm{T}$ and $\mathrm{D}$ denote Chandler, tropical and draconitic oscillations, respectively. Residuals $\mathbf{r}(t)$ that are marked as a sum of Common Mode Errors CME $(t)$ and noise $\boldsymbol{\varepsilon}(t)$, are subject of further analysis. This equation was modified following Bogusz and Klos (2015) findings, who emphasized the significance of above mentioned periodic signals, when one performs noise analysis.

In this research we adopted a general spatiotemporal filtering approach that uses an orthogonal transformation named Principal Component Analysis (PCA). This statistical procedure is based on a full observation matrix. In our case, the observation matrix is constructed from residual time series $\mathbf{r}(t)$. Initially, we assumed that our time series are regular and continuous. We formed an observation matrix $\mathbf{R}\left(t_{i}, r_{j}\right)(i=1,2, \ldots, m$ and $j=1,2, \ldots, n)$, using a GNSS station network formed by $n$ stations, with time series spanning $m$ days. We created this matrix for each topocentric component (North, East and Up) separately. Each row refers to a specified, subsequent, equally sampled observation epoch, while each of the randomly arranged columns is equivalent to the $j$-th station residual time series. In consequence, based on the elements of the observation matrix, each element of the covariance $(n \times n)$ symmetric matrix $\mathbf{B}\left(t_{i}, r_{j}\right)$ is defined as (Dong et al., 2006):

$$
b_{i j}=\frac{1}{m-1} \sum_{k=1}^{m} \mathbf{R}\left(t_{k}, r_{i}\right) \mathbf{R}\left(t_{k}, r_{j}\right)
$$

PCA is performed by eigenvalue decomposition of a data covariance matrix $\mathbf{B}$ into the eigenvector matrix $\mathbf{V}^{T}$ that is a $(n \times n)$ matrix with orthonormal rows, and the $\boldsymbol{\Lambda}$ matrix, which has $k$ nonzero diagonal eigenvalues $\left\{\lambda_{k}\right\}(k \geq n)$ (Jolliffe, 2002):

$$
\mathbf{B}=\mathbf{V} \boldsymbol{\Lambda} \mathbf{V}^{T}
$$

A decreasing order of eigenvalues and corresponding columns in eigenvector matrix is needed to define principal components (Jolliffe, 2002): 
$\mathbf{a}_{k}\left(t_{i}\right)=\sum_{j=1}^{n} \mathbf{R}\left(t_{i}, r_{j}\right) \mathbf{v}_{k}\left(r_{j}\right)$

where $a_{k}\left(t_{i}\right)$ [in the left side symbol $\mathbf{a}_{\mathrm{k}}$ should be bold text but no italic (like it is in above formula)] is $k$-th principal component of matrix $\mathbf{R}$ and $\mathbf{v}_{k}$ is corresponding eigenvector. The resulting orthogonal components (Principal Components) are ordered by the magnitude of variation, from most to least. First few components are a product of a uniform temporal function (CME) (Jolliffe, 2002).

In order to express eigenvector elements more transparently (as a station's spatial response to a CME source) we divided each element in an eigenvector by maximal value from this eigenvector. In all cases, one of the elements of eigenvector will have a $100 \%$ response, while a percentage of remaining elements will refer to this largest value. Each element of eigenvector, and eigenvector itself, is calculated this way, expressed as a percentage, and is labeled as a "normalized response" and "normalized eigenvector," respectively (Dong et al., 2006).

The method of deriving CME from PCs is not clearly defined. There are approaches that use statistical tests to determine, which PCs are significant in terms of variance accounted for by each component (Tiampo et al., 2004; Shen et al., 2013). We adopted the CME definition proposed in the paper by Dong et al. (2006), which is the most conventional and intuitive. This allows us to avoid consequences of incorrectly identified, statistically significant principal components. The basis of these assumptions is to select a principal component, for which more than half corresponding normalized responses have a value larger than $25 \%$. An eigenvalue corresponding to this $\mathrm{PC}$, has to be larger than $1 \%$ of the sum of all eigenvalues. Using this method, we found $p$ first numbers of significant PCs, and we then computed CME as follows (Dong et al., 2006):

$$
C M E_{j}\left(t_{i}\right)=\sum_{k=1}^{p} \mathbf{a}_{k}\left(t_{i}\right) \mathbf{v}_{k}\left(r_{j}\right)
$$

The computed CME is removed from the unfiltered residuals $\mathbf{r}(t)$ using simple subtraction, thus obtaining so-called "filtered residuals." This approach is equivalent to the reconstruction of the filtered residual using all PCs, except for the $p$ first significant PCs.

\section{ANALYSIS AND RESULTS}

In order to give a better visual representation of common signal strength in the residual time series, we show in Figure 2 eigenvalues referring to each consecutive principal component. We express the eigenvalues as a percentage of the total variance in the dataset. These are related to the amount of variation in the entire data set, explained by the corresponding PC
(Jolliffe, 2002). We can see, that first principal component computed for North explains nearly $40 \%$ of the variation, while the first PC for East and Up accounts for more than half of the variation (Fig. 2).

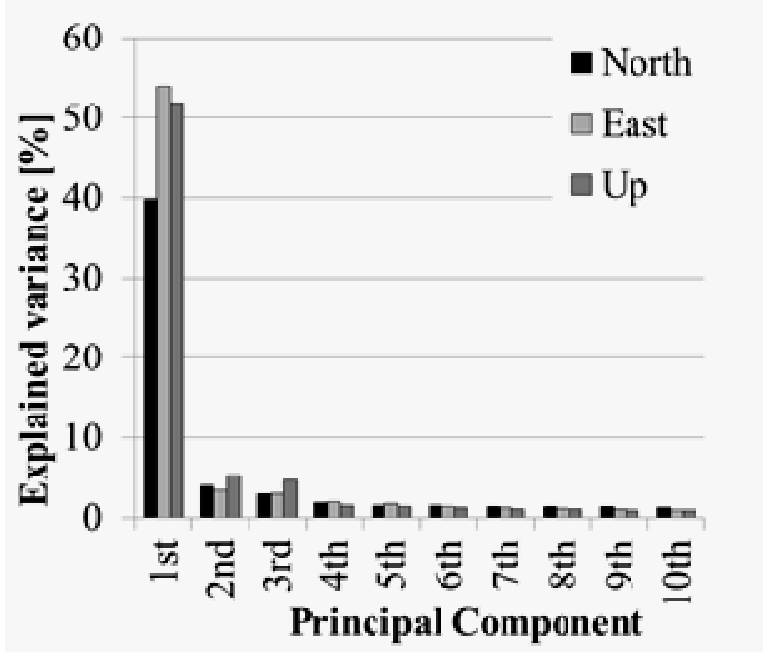

Fig. 2 Eigenvalue expressed as proportion of the variance that each eigenvector represents. Only 10 first PCs are presented.

Then, we selected components that may be identified as CME, using previously described criterion. For each topocentric components, all normalized spatial responses corresponding to the $1^{\text {st }}$ PC are larger than $25 \%$. This satisfies PC requirements in respect to its inclusion into $\mathrm{CME}$ determination. The number of significant eigenvector elements is much lower when $2^{\text {nd }} \mathrm{PC}$ is considered. From the 87 element sized eigenvector, there were 9, 3 and 31 normalized responses to the $2^{\text {nd }} \mathrm{PC}$ greater than $25 \%$ for North, East and Up, respectively. The $2^{\text {nd }}$ and each following PC are not sufficient to calculate CME. The $2^{\text {nd }} \mathrm{PC}$ computed for Up, was the closest to being classified as significant, but was rejected due to the fact that it had only around $50 \%$ significant responses. Spatial distribution of normalized eigenvector elements corresponding to the $1^{\text {st }}$ and $2^{\text {nd }}$ PC computed for Up component is presented in Figure 3. On the left side map, we can see that all stations have a positive response to the $1^{\text {st }} \mathrm{PC}$ larger than $30 \%$. It is confirmed that in our case the $1^{\text {st }} \mathrm{PC}$ reflects Common Mode Error that is the effect of a uniform temporal function.

In Figure 3 on the right side map we can see normalized spatial responses for the $2^{\text {nd }} \mathrm{PC}$ computed for Up component. The largest, $100 \%$ response to this PC's variation has VLUC (Vallodella Lucania, Italy) station. We can see that in this case, the response to the $2^{\text {nd }} \mathrm{PC}$ is getting smaller when a station is located farther from this station, when with a specified distance response changes to negative. Such an effect refers to smaller spatial scale common signals in network that can be seen in higher-order PCs and is difficult to be interpreted. 
In order to identify the filtration benefits, we computed power-law noise amplitudes and spectral indices of noise for residuals before and after filtering. If we hypothetically assume a lack of CME, and if residuals would only be a realization of stochastic processes, the spectral index should be close to 0 (white noise). In reality, the spectral indices computed for residual GNSS time series are fractional numbers lower than zero, which indicates that some kind of colored noise should be considered during spectral analysis (e.g. Mao et al., 1999; Agnew, 1992; Williams, 2003; Beavan, 2005; Amiri-Simkooei et al., 2007; Bos et al., 2008; Langbein, 2008; Teferle et al., 2008; Kenyeres and Bruyninx, 2009; SantamaríaGómez et al., 2011 or Klos et al., 2016). In order to perform this analysis, we used Hector software (Bos et al., 2008). The values of spectral indices computed before filtration for 87 stations are presented in Table 1.

Table 1 Spectral indices computed for 87 residual time series before filtration.

\begin{tabular}{lccc}
\hline & North & East & Up \\
\hline median & -0.60 & -0.48 & -0.66 \\
$\min$ & -0.96 & -0.67 & -1.00 \\
$\max$ & -0.15 & -0.23 & -0.19 \\
\hline
\end{tabular}

Spatial filtration of residuals with an assumption of a uniform temporal function across the network, should shift spectral indices toward white noise, and reduce amplitudes. Previous applications of PCA to GNSS time series (Dong et al., 2006; Shen et al., 2013) assumed models consisting of only annual and semi-annual oscillations. It should confirm that most common time-dependent signals affecting residuals, and lead to spatial correlation in horizontal components, had been modeled by us earlier with a higher efficiency model (Bogusz and Klos, 2015). Mean, median, maximal and minimal value of powerlaw noise amplitudes computed for Up component are equal to $12.0,11.7,6.2$ and $21.2 \mathrm{~mm} / \mathrm{year}^{-\mathrm{k} / 4}$, respectively. Figure 4 presents power-law noise amplitudes and spectral indices changes after filtration computed for residuals of Up component. A reduction of these parameters for horizontal components is not evident probably due to lesser correlation.

From top histogram in Figure 4 we can notice, that for all residual time series of $\mathrm{Up}$ component, noise amplitudes decreased from 0.5 to $13.5 \mathrm{~mm} /$ year $^{-\mathrm{k} / 4}$ after filtration. That gives a relative reduction of amplitudes ranging between $4 \%$ and $76 \%$ for all stations, while the average improvement is $49 \%$. A smaller amplitude of noise after filtration means smaller scatter of residuals, and consequently, a more stable solution for the determination of station velocity. The bottom histogram refers to spectral

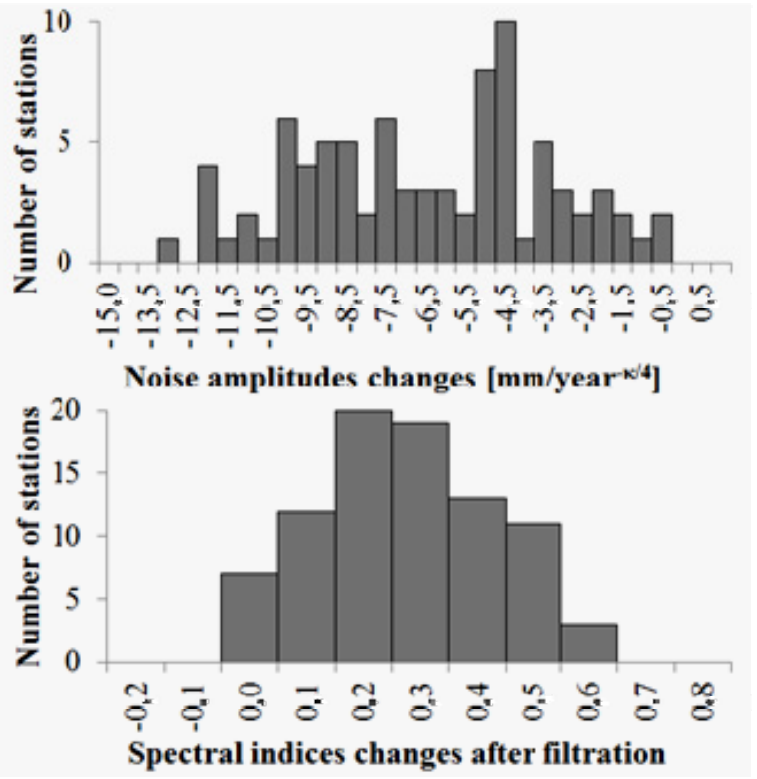

Fig. 4 Histograms of noise amplitudes (top) and spectral indices (bottom) changes after filtration of $\mathrm{Up}$ component residual time series for 87 stations.

indices changes after filtration. We can see positive changes (increase), which are equivalent to a shift of stochastic part towards white noise. Furthermore, the positive result of filtering means that correlated signals were superimposed on the stochastic process realization. The average relative increase of the spectral index is $48 \%$.

One of the most important consequences related to spatio-temporal GNSS time series filtering is the improvement of accuracy of the determined velocity. Assuming that the station velocity can be determined using linear regression (trend is strictly linear), we can compute the accuracy of velocity using the formula presented in the paper by Bos et al. (2008), which is based on values of noise characteristics:

$$
m_{v} \approx \pm \sqrt{\frac{A_{P L}^{2}}{\Delta T^{2-\frac{\kappa}{2}} \cdot \frac{\Gamma(3-\kappa) \cdot \Gamma(4-\kappa) \cdot(N-1)^{\kappa-3}}{\left[\Gamma\left(2-\frac{\kappa}{2}\right)\right]^{2}}}}
$$

where: $N$ is the data length, $\kappa$ means the estimated spectral index, $\Delta T$ is the sampling rate, $A_{P L}$ represents the amplitude of noise, and $\Gamma$ is the gamma function.

The improvement of the velocity accuracy, resulting from the filtration computed for $\mathrm{Up}$ component, for the 87 stations being analyzed, is presented in Figure 5.

For Up component of all stations, the accuracy of velocity is lower after filtration. The average reduction is $0.2 \mathrm{~mm} /$ year, with maximal reduction is $0.8 \mathrm{~mm} /$ year determined for BUDP (Kobenhavn, 


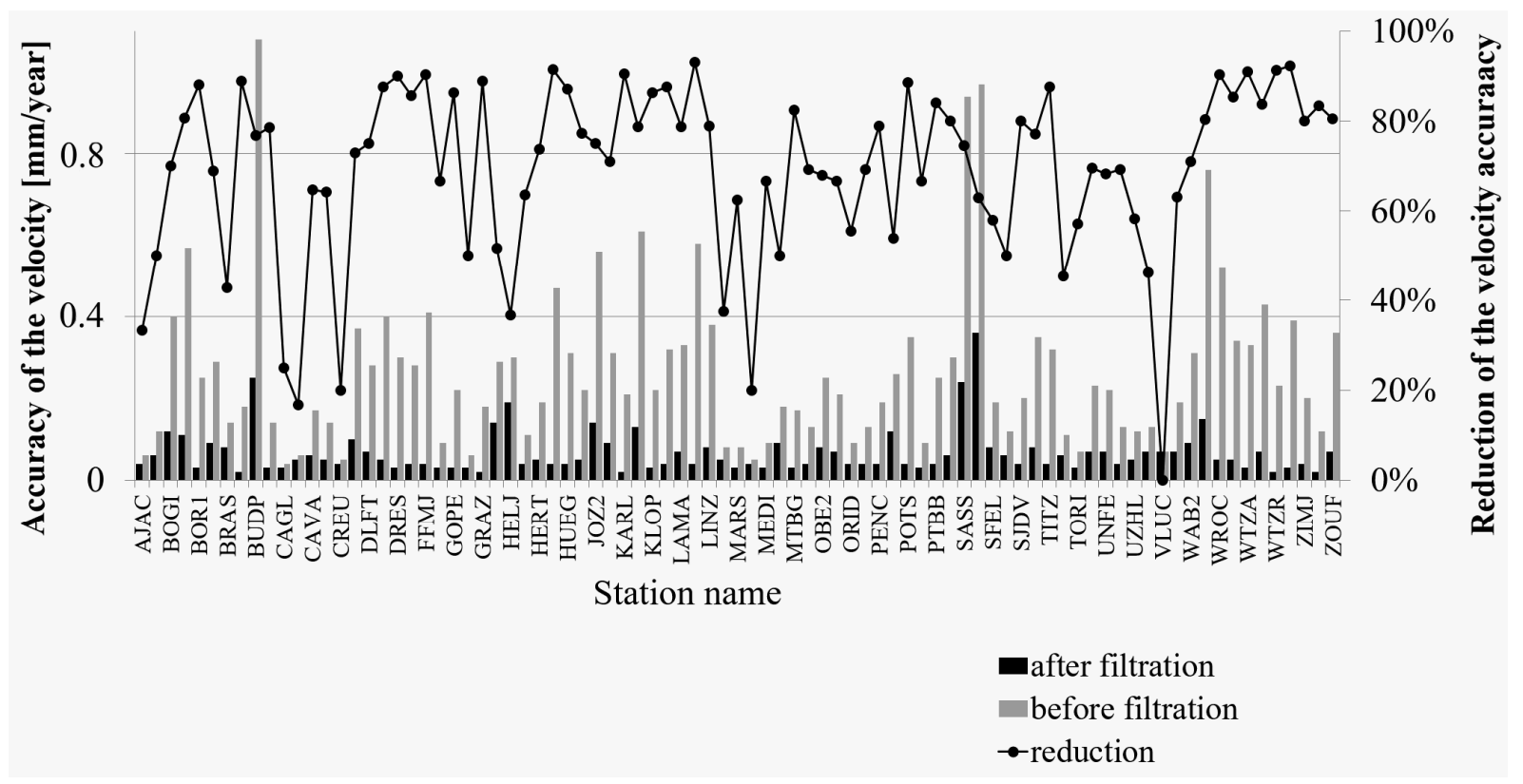

Fig. 5 Reduction of determined velocity accuracy resulting from the PCA-based method filtration. Bars refer to the left side axis and show the accuracy of the velocity computed before the subtraction of CME (gray) and after filtration (black). The line refers to the right side axis and explains the relative reduction of accuracy resulting from spatio-temporal filtering.

Denmark) station. The above described result means that the after-filtration reduction of accuracy is on average about $70 \%$ on average.

\section{SUMMARY}

Orthogonal transformation of the covariance matrix leads us to obtain linearly uncorrelated variables that are sorted by the amount of variance. Taking into account residual time series from stations located in the regional network, and adopting appropriate assumptions, we can separate significant signals from this network. Correlated signals named Common Mode Error, are effects of the existence of uniform temporal function across the network. We noticed that for an analyzed network, only the first PC mode is a suitable candidate for CME, because the high-order modes are usually related to a few stations, and reflect week local effects. Due to the weaker spatial correlation in horizontal components, this method appears to be efficient only for vertical changes. Results confirm, that spatio-temporal filtration gives significant changes in noise characteristics, and velocity accuracy deterioration when Up component is considered. The above explained effect result in greater precision and reliability of the time series used for geodynamic studies.

\section{ACKNOWLEDGMENTS}

This research was supported by the Military University of Technology, Faculty of Civil Engineering and Geodesy Young Scientists Development funds (739/2015).
JPL repro2011b time series accessed from ftp://sideshow.jpl.nasa.gov/pub/JPL_GPS Timeseries/ repro2011b/raw/ on 2014-11-10.

Map was drawn in the GMT (Generic Mapping Tool) (Wessel et al., 2013).

\section{REFERENCES}

Agnew, D.C.: 1992, The time-domain behaviour of powerlaw noises. Geophys. Res. Lett., 19, No. 4, 333-336. DOI: 10.1029/91GL02832

Amiri-Simkooei, A.R., Tiberius, C.C.J.M. and Teunissen, P.J.G.: 2007, Assessment of noise in GPS coordinate time series: Methodology and results. J. Geophys. Res., 112, B07413. DOI: 10.1029/2006JB004913

Amiri-Simkooei, A. R.: 2013, On the nature of GPS draconitic year periodic pattern in multivariate position time series. J. Geophys. Res., Solid Earth, 118, 2500-2511. DOI: 10.1002/jgrb.50199

Beavan, J.: 2005, Noise properties of continuous GPS data from concrete pillar geodetic monuments in New Zealand and comparison with data from U.S. deep drilled braced monuments. J. Geophys. Res., 110, B08410. DOI: 10.1029/2005JB003642

Bogusz, J., Gruszczynski, M., Figurski, M. and Klos, A.: 2015, Spatio-temporal filtering for determination of common mode error in regional GNSS networks. Open Geosciences, 7, No. 1, 140-148. DOI: $10.1515 /$ geo-2015-0021

Bogusz, J. and Klos, A.: 2015, On the significance of periodic signals in noise analysis of GPS station coordinates time series. GPS Solutions. DOI: $10.1007 / \mathrm{s} 10291-015-0478-9$

Bos, M.S., Fernandes, R.M.S., Williams, S.D.P. and Bastos, L.: 2008, Fast error analysis of continuous GPS observations. J. Geod., 82, No. 3, 157-166. DOI: $10.1007 / \mathrm{s} 00190-007-0165-\mathrm{x}$ 
Chang, E.T.Y. and Chao, B.F.: 2014, Analysis of coseismic deformation using EOF method on dense, continuous GPS data in Taiwan. Tectonophysics, 637, 106-115. DOI: 10.1016/j.tecto.2014.09.011

Dong, D., Fang, P., Bock, Y., Webb, F., Prawirodirdjo, L., Kedar, S. and Jamason, P.: 2006, Spatio-temporal filtering using principal component analysis and Karhunen-Loeve expansion approaches for regional GPS network analysis. J. Geophys. Res., 111, B03405. DOI: $10.1029 / 2005 J B 003806$

He, X., Hua, X., Yu, K., Xua, W., Lu, T., Zhang, W. and Chen, X.: 2015, Accuracy enhancement of GPS time series using principal component analysis and block spatial filtering. Advances in Space Research, 55, No. 5, 1316-1327. DOI: 10.1016/j.asr.2014.12.016

Jackson, J.E.: 1991, A users guide to principal components. Wiley, NewYork. DOI: 10.1002/0471725331

Jolliffe, I.T.: 2002, Principal component analysis. Springer Verlag, New York. DOI: 10.1007/b98835

Kenyeres, A. and Bruyninx, C.: 2009, Noise and periodic terms in the EPN time series. Geodetic Reference Frames. International Association of Geodesy Symposia, 134, 143-148. DOI: 10.1007/978-3-642-00860-3_22

Klos, A., Bogusz, J., Figurski, M. and Gruszczyński, M.: 2016, Error analysis for European IGS stations. Stud. Geophys. Geod., 60, 17-34. DOI: $10.1007 / \mathrm{s} 11200-015-0828-7$

Langbein, J.: 2008, Noise in GPS displacement measurements from Southern California and Southern Nevada. J. Geophys. Res., 113, B05405. DOI: 10.1029/2007JB005247

Li, W., Shen, Y. and Li, B.: 2015, Weighted spatio-temporal filtering using principal component analysis for analyzing regional GNSS position time series. Acta Geodaet. Geophys., 50, No. 4, 419-436. DOI: $10.1007 / \mathrm{s} 40328-015-0100-1$

Liu, H., Shah, S. and Jiang, W.: 2004, On-line outlier detection and data cleaning, Computers and Chemical Engineering, 28, 1635-1647.

DOI: 10.1016/j.compchemeng.2004.01.009

Mao, A., Harrision, C. and Dixon, T.: 1999, Noise in GPS coordinate time series. J. Geophys. Res., 104 (B2), 2797-2816. DOI: 10.1029/1998JB900033

Márquez-Azúa, B. and DeMets, C.: 2003, Crustal velocity field of Mexico from continuous GPS measurements. 1993 to June 2001: Implications for the neotectonics of Mexico. J. Geophys. Res., 108(B9), 2450. DOI: 10.1029/2002JB002241

Nikolaidis, R.: 2002, Observation of geodetic and seismic deformation with the Global Positioning System. Ph.D. thesis, Univ. of Calif., San Diego.

Rebischung, P., Griffiths, J., Ray, J., Schmid, R., Collilieux, X., and Garayt, B.: 2012, IGS08: the IGS realization of ITRF2008. GPS Solut, 16, 483-494. DOI 10.1007/s10291-011-0248-2

Rodionov, S. and Overland, J.E.: 2005, Application of a sequential regime shift detection method to the Bering Sea ecosystem. ICES Journal of Marine Science, 62, 328-332. DOI: 10.1016/j.icesjms.2005.01.013

Santamaría-Gómez, A., Bouin, M.-N., Collilieux, X. and Wöppelman, G.: 2011, Correlated errors in GPS position time series: Implications for velocity estimates. J. Geophys. Res., 116, B01405.

DOI: $1.0 .1029 / 2010 J B 007701$
Savage J.: 1995, Principal component analysis of interseismic deformation in southern California. J. Geophys. Res., 100, No. 12, 691-701.

Shen, Y., Li, W., Xu, G. and Li, B.: 2013, Spatio-temporal filtering of regional GNSS network's position time series with missing data using principle component analysis. J. Geod., 88, 351-360. DOI: 10.1007/s00190-013-0663-y

Teferle, F. N., Bingley, R. M., Dodson, A. H., Penna, N. T. and Baker, T.F.: 2002, Using GPS to separate crustal movements and sea level changes at tide gauges in the UK. In: Vertical Reference Systems, edited by H. Drewes, et al., Springer-Verlag, Heidelberg Berlin, 264-269.

Teferle, F.N., Williams, S.D.P., Kierulf, K.P., Bingley, R.M. and Plag, H.P.: 2008, A continuous GPS coordinate time series analysis strategy for high-accuracy vertical land movements. Physics and Chemistry of the Earth, 33, 205-216. DOI: 10.1016/j.pce.2006.11.002

Tiampo, K.F., Rundle, J.B., Klein, W., Ben-Zion, Y. and McGinnis, S.: 2004, Using eigenpattern analysis to constrain seasonal signals in southern California. Pure Appl. Geophys., 161, No. 9, 1991-2003.

DOI: $10.1007 / \mathrm{s} 00024-004-2545-\mathrm{y}$

Tian, Y. and Shen, Z.K.: 2011, Correlation weighted stacking filtering of common-mode component in GPS observation network. Acta Seismol. Sin., 33, No. 2, 198-208.

Tian, Y. and Shen, Z.K.: 2016, Extracting the regional common-mode component of GPS station position time series from dense continuous network. J. Geophys. Res., Solid Earth. DOI: 10.1002/2015JB012253

Wdowinski, S., Bock, Y., Zhang, J., Fang, P. and Genrich, J.: 1997, Southern California permanent GPS geodetic array: Spatial filtering of daily positions for estimating coseismic and postseismic displacements induced by the 1992 Landers earthquake. J. Geophys. Res., 102(B8), 18057-18070. DOI: 10.1029/97JB01378

Wessel, P., Smith, W.H.F., Scharroo, R., Luis, J. and Wobbe, F.: 2013, Generic Mapping Tools: Improved Version Released. Eos, Transactions, American Geophysical Union, 94, No. 45, 409-410. DOI: $10.1002 / 2013 \mathrm{EO} 450001$

Williams, S.D.P.: 2003, The effect of coloured noise on the uncertainties of rates estimated from geodetic time series. J. Geod., 76, 483-494. DOI 10.1007/s00190-002-0283-4

Williams, S.D.P., Bock, Y., Fang, P., Jamason, P., Nikolaidis, R.M., Prawirodirdjo, L., Miller, M. and Johnson, D.J.: 2004, Error analysis of continuous GPS position time series. J. Geophys. Res., 109, B03412. DOI: 10.1029/2003JB002741

Witchayangkoon, B.: 2000, Elements of GPS Precise Point Positioning. Ph.D. thesis, University of Maine. 

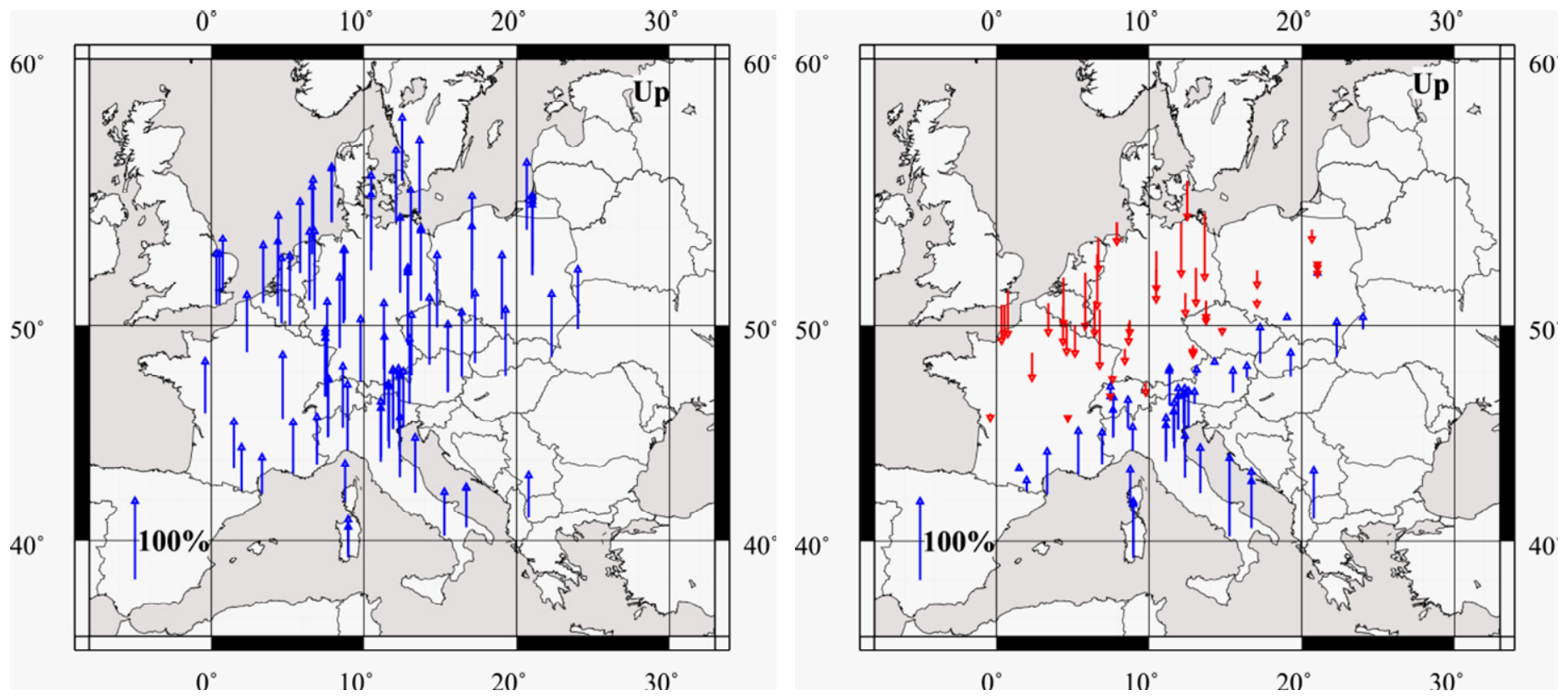

Fig. 3 Spatial distribution of the normalized responses (normalized eigenvector elements) to the $1^{\text {st }}$ (left) and $2^{\text {nd }}$ (right) PC for Up component. Normalized response can be identified with station contribution (positive-blue or negative-red) into the amount of variation in particular PC. 